\title{
Variable Winds in Early-B Hypergiants
}

\author{
Bernhard Wolf and Thomas Rivinius ${ }^{1}$ \\ Landessternwarte Königstuhl, D-69117 Heidelberg, Germany
}

\begin{abstract}
Early-B hypergiants belong to the most luminous stars in the Universe. They are characterized by high mass-loss rates $\left(\dot{M} \approx 10^{-5} \mathrm{M}_{\odot} \mathrm{yr}^{-1}\right)$ and low terminal wind velocities $\left(v_{\infty} \approx 400 \mathrm{~km} \mathrm{~s}^{-1}\right)$ implying very dense winds. They represent a short-lived evolutionary phase and are of particular interest for evolutionary theories of massive stars with mass loss. Due to their high luminosity they play a key role in connection with the "wind momentum - luminosity relation". Among the main interesting characteristics of early-B hypergiants are the various kinds of photometric and spectroscopic variations. In several recent campaigns our group has performed extensive high dispersion spectroscopy of galactic early-B hypergiants with our fiber-fed echelle spectrograph FLASH/HEROS at the ESO-50 cm telescope. The main outcome was that their dense winds behave hydrodynamically differently to the less luminous supergiants of comparable spectral type. Outwardly accelerated propagating discrete absorption components of the P Cyg-type lines are the typical features rather than rotationally modulated line profile variations. These discrete absorptions could be traced in different spectral lines from photospheric velocities up to $75 \%$ of the terminal velocity. The stellar absorption lines show a pulsation-like radial velocity variability pattern lasting up to two weeks as the typical time scale. The radius variations connected with this pulsation-like motions are correlated with the emission height of the P Cyg-type profiles.
\end{abstract}

\section{Main Properties and Variability of Early-B Hypergiants}

Early B-hypergiants belong to the most luminous stars in the Universe $\left(M_{\text {bol }} \approx\right.$ $-10 \ldots-11$ ) with effective temperatures around $20000 \mathrm{~K}$. They represent short-lived evolutionary phases of massive stars. For the given luminosity the masses are comparatively low. In this connection R81 (B2.5Ia-O) is of particular interest; it is an eclipsing binary (Wolf et al. 1981, Stahl et al. 1987). A rough estimate from the light curve gives $33 \mathrm{M}_{\odot}$, i.e. $\mathrm{R} 81$ has presumably lost more than half of its initial mass in previous evolutionary phases.

IUE observations have shown that early-B hypergiants are characterized by high mass-loss rates $\left(\dot{M} \approx 10^{-5} \mathrm{M}_{\odot} \mathrm{y}^{-1}\right)$ with comparatively low velocities $\left(v_{\infty} \approx 400 \mathrm{~km} \mathrm{~s}^{-1}\right)$ implying rather high wind densities. In addition to the usual wind indicators numerous lines originating from metastable states formed in the wind (particularly FeIII lines) were identified (cf. e.g. Wolf \& Appenzeller 1979, Appenzeller \& Wolf 1979, Prinja et al. 1990, Lamers et al. 1995). 


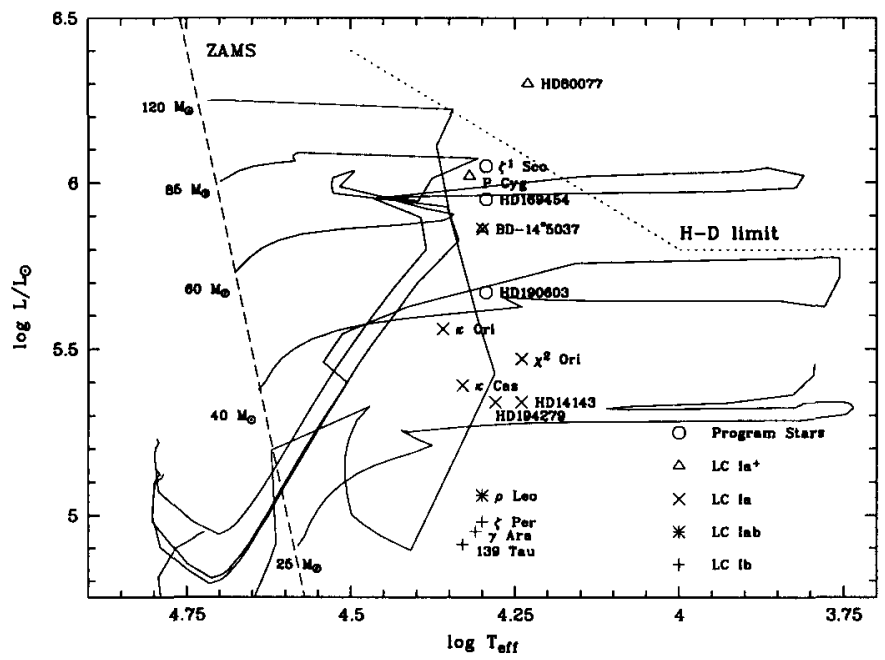

Fig. 1. A sample of early B super- and hypergiants shown in the Hertzsprung Russell diagram

Due to these characteristics early-B hypergiants are important test cases for:

a) evolutionary theories of massive stars with mass loss (cf. e.g. Schaerer et al. 1996)

b) the "wind momentum - luminosity relation" (high luminosity) (cf. e.g. Kudritzki, this Volume)

c) the "bi-stability jump" of $v_{\infty} / v_{\text {esc }}$ of 2.6 at $T_{\text {eff }}>21000$ to 1.3 at $T_{\text {eff }}<$ 21000 (Lamers et al. 1995).

Photometric variations of the prototype $\zeta^{1}$ Sco in time scales of 11 to 16 days of about $0.1 \mathrm{mag}$ were reported by Sterken (1977) and Burki et al. (1982). Spectroscopic snap-shot observations of early-B hypergiants in the optical range have shown pronounced line-profile variations of the P Cygnitype lines and radial velocity variations of the photospheric absorption lines with amplitudes of about $20 \mathrm{~km} \mathrm{~s}^{-1}$. The photospheric absorption lines are asymmetric with blue wings extending to -200 and $-300 \mathrm{~km} \mathrm{~s}^{-1}$ (Sterken \& Wolf, 1978,1979). From various IUE observations profile variations of wind lines with variable wind absorption components were discovered (Burki et al. 1982). These observations indicate:

a) a pure hydrostatic photosphere does not exist; time and depth dependent velocity fields in the atmosphere are the predominant features.

b) considerable mass-loss variations do occur.

What is the nature of these variations? What kind of hydrodynamic processes, pulsation-like motions etc. are prevailing? Are there obvious corre- 
Table 1. Stellar parameters of the observed stars

\begin{tabular}{lr|ccc} 
Object & & $\zeta^{1}$ Sco & HD 169454 & HD 190603 \\
\hline$T_{\text {eff }}$ & {$[\mathrm{K}]$} & 19700 & 19700 & 19700 \\
$\log g$ & & 2.30 & 2.30 & 2.40 \\
\hline$R_{\star}$ & {$\left[\mathrm{R}_{\odot}\right]$} & 91 & 82 & 59 \\
$M_{\star, \log g}$ & {$\left[\mathrm{M}_{\odot}\right]$} & 60 & 48 & 32 \\
$M_{\star, \text { evol. track }}$ & {$\left[\mathrm{M}_{\odot}\right]$} & 40 & 40 & 32 \\
$\log L_{\star} / \mathrm{L}_{\odot}$ & & 6.05 & 5.95 & 5.67 \\
$M_{\mathrm{V}}$ & {$[\mathrm{mag}]$} & -9.09 & -8.85 & -8.03 \\
\hline$\dot{M}$ & {$\left[10^{-6} \mathrm{M}_{\odot} \mathrm{yr}^{-1}\right]$} & 6.2 & 6.3 & 2.7 \\
$v_{\infty}$ & {$\left[\mathrm{km} \mathrm{s}^{-1}\right]$} & 370 & 483 & 515 \\
$v_{\text {esc }}$ & {$\left[\mathrm{km} \mathrm{s}^{-1}\right]$} & 365 & 345 & 360
\end{tabular}

lations of the wind lines' variability and the variations of the photospheric absorption lines? And how do the B hypergiants compare with the less luminous B supergiants?

To learn more about the atmospheric motions and wind properties of early B hypergiants, snap shot observations are not sufficient - extended spectroscopic campaigns are a prerequisite. Such campaigns were carried out by us with Flash/Heros (cf. Rivinius et al. 1997, Kaufer,1998 and this Volume) at the ESO- $50 \mathrm{~cm}$ telescope. The typical stellar parameters of the target stars are given in Table 1 and their location in the HRD is shown by Figure 1. The dates of the observational campaigns are given in Table 2.

\section{Line-Profile and Radial Velocity Variations}

Our high dispersion spectroscopic campaigns allowed us to follow the wind properties and teir variability down to very deep layers and to diagnose the photosphere-wind interface of early-B hypergiants. We will briefly demonstrate that for the case of the prototype $\zeta^{1}$ Sco.

Table 2. The observation campaigns

\begin{tabular}{|c|c|c|c|c|c|c|c|}
\hline \multirow[b]{2}{*}{ Object } & \multicolumn{6}{|c|}{ Spectra/Nights } & \multirow{2}{*}{$\begin{array}{c}\text { Ave. } S / N \\
{[5350 \AA-} \\
5450 \AA]\end{array}$} \\
\hline & 1990 & 1991 & 1992 & 1993 & 1994 & 1995 & \\
\hline$D$ & $28 / 87$ & $42 / 154$ & - & - & - & - & 330 \\
\hline$\zeta^{1} \mathrm{Scc}$ & - & - & $37 / 52$ & $93 / 114$ & $103 / 128$ & $57 / 120$ & 110 \\
\hline HD 169454 & - & - & $14 / 45$ & $11 / 17$ & $25 / 62$ & $2 / 2$ & 100 \\
\hline
\end{tabular}



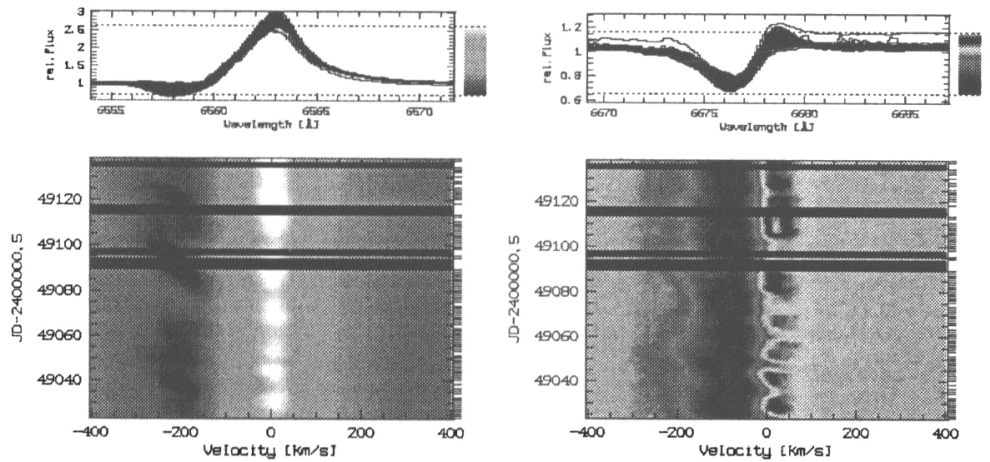

Fig. 2. The dynamical spectra of $\mathrm{H} \alpha$ and HeI $\lambda 6678$ in 1993
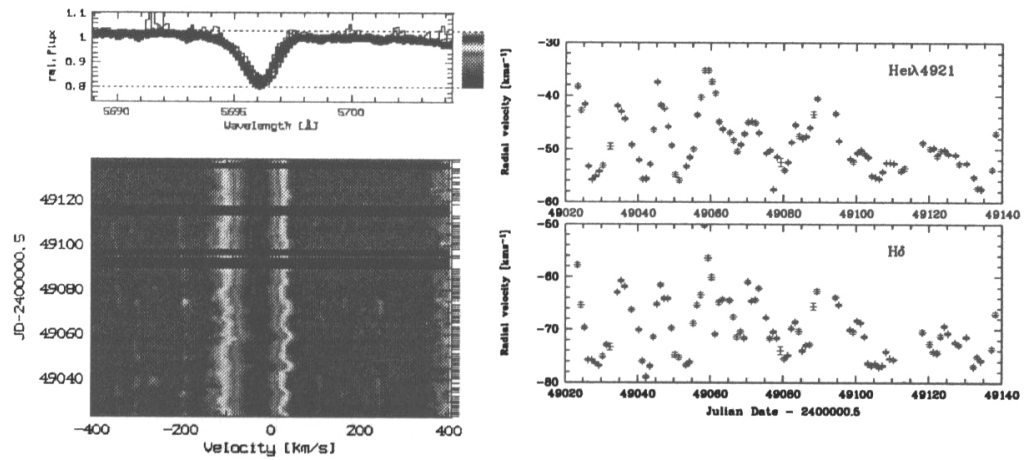

Fig. 3. The dynamical spectrum of AlIII $\lambda 5696$ and the measured radial velocities of $\mathrm{HeI} \lambda 4921$ and $\mathrm{H} \delta$ in 1993

\subsection{Observations}

Fig. 2 shows the dynamical spectra of $\mathrm{H} \alpha$ and of Hie $\lambda 6678$ of $\zeta^{1}$ Sco as observed during the 1993 campaign. As shown by the figure the P Cyg profiles are characterized by accelerated absorption features formed throughout the wind. One has immediately the impression, that this time series is a key finding to investigate the wind variation and that it opens for the first time the chance to derive empirically the velocity law of wind features. There is no obvious connection between emission and absorption variability in the $\mathrm{P}$ Cyg profiles; the repetition time of the absorption features is about 24 days. The emission variability is similar in all P Cyg lines; the repetition time is about 15 days and is comparable to the time scale of the photometric variability.

The kind of radial velocity variations of photospheric lines is shown by Fig. 3. It contains the dynamical spectrum of the line Alın $\lambda 5696$ and the plots of the variations of the photospheric absorption lines HeI $\lambda 4921$ and $\mathrm{H} \delta$. Cyclical variability is present most of the time, the typical cycle times being 10 to 20 days. After several weeks it eventually evolves into icoherent vari- 

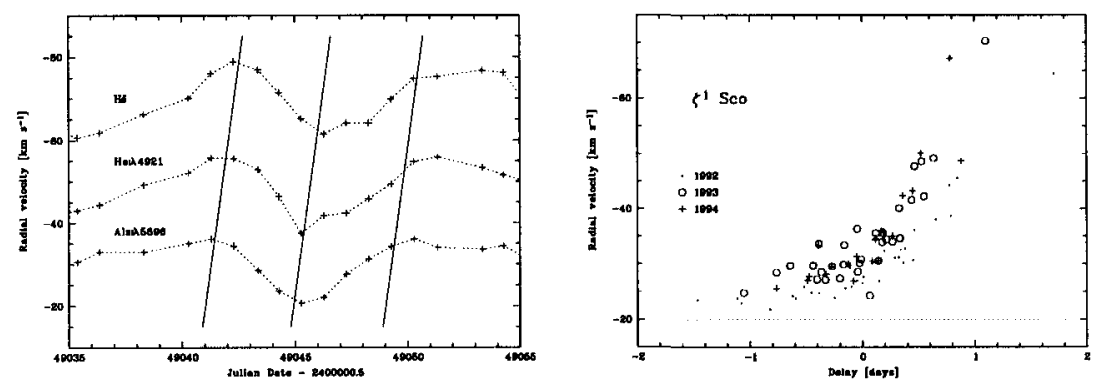

Fig. 4. The temporal shifts of the variation pattern for lines originating in different depths of the photoosphere (left) and the derived pattern delays of different lines plotted versus their mean velocities
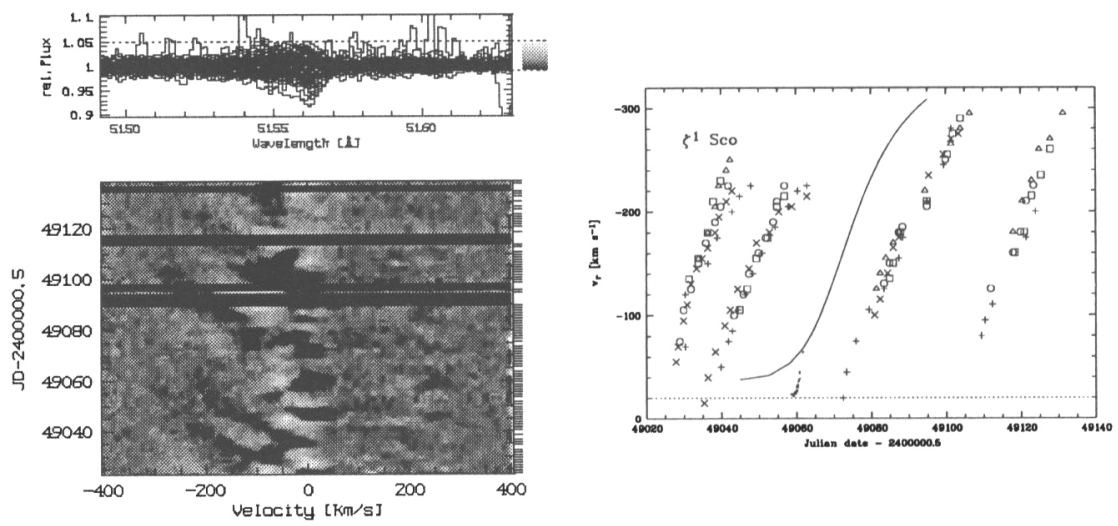

Fig. 5. Dynamical residual spectrum of FeIII $\lambda 5156$ in 1993 and the measured velocity increase of propagating disturbances. Also shown is a $\beta$ type velocity law with $\beta=2.5$. The dots connecting to the $\beta$-law are the values given in Fig. 4 (right)

ability. Pulsation-like motions are evident.(Note that the early-B type hypergiants are located in the HRD in a region where Kiriakidis et al. (1993) have predicted strange mode oscillations). The pulsation-like variability pattern is similar in all lines, but the average velocity depends on the line strength, i.e. the weekest lines show the smallest velocities. Moreover, as shown by Fig.4 the variability of lines of higher velocities (i.e. of lines formed further out in the photosphere) is delayed by up to three days; the delay can be determined by cross correlation. A model comes up that the acceleration of the disturbances has something to do with the pulsation-like motions. 

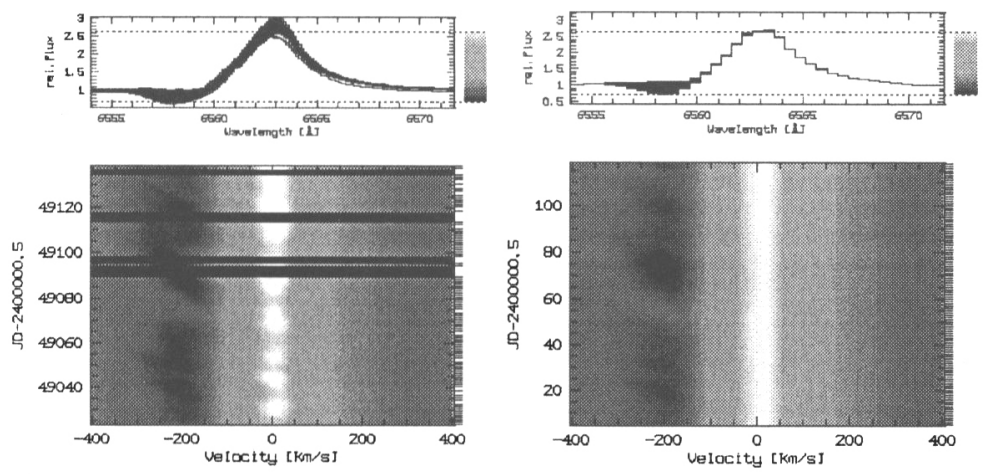

Fig. 6. The dynamical spectra of $\mathrm{H} \alpha$ and the modelled variability in 1993

\subsection{Velocity Law of Perturbations}

The dynamical spectra of $\mathrm{H} \alpha$ (cf. Fig.2) and $\mathrm{H} \beta$ were used to diagnose the propagation of disturbances in the outer part through the wind. In order to trace back the velocity law to photospheric velocities we searched for fainter P Cyg-type profiles in which the absorption features can be traced down to low velocities. The FeIII $\lambda \lambda 5127,5156$ lines (cf. Fig.5) have these properties. Four events are visible in these lines in 1993. If we trace these events we get the velocity curves which can be compared with $\beta$-type velocity laws (Fig.5). $\beta=2.5$ provides a reasonable description, but note that the acceleration of the disturbances is much steeper close to the photosphere than any $\beta$ law, which are mostly used to describe the winds of hot stars (cf. e.g. Rivinius et. al., 1997) In addition the pulsation-like motions and the delay of the radialvelocity pattern for photospheric lines formed at different depths can be used to diagnose the photosphere-wind interface. As indicated by the diagram in Fig. 5 the same steep acceleration is indicated by this delay pattern.

To our knowledge this is for the first time that the velocity law has been directly derived from the propagation of wind features in B supergiants. But

Table 3. Model parameters for the observed stars. The temperature distribution in the wind is given by a square root law

\begin{tabular}{|c|c|c|c|}
\hline Object & $\zeta^{1}$ Sco & HD 169454 & HD 190603 \\
\hline$\left[\mathrm{km} \mathrm{s}^{-1}\right]$ & 18 & 18 & 18 \\
\hline$\beta$ & 1.7 & 1.5 & 1.7 \\
\hline$v_{\text {turb }} / v_{\infty}$ & 0.05 & 0.037 & 0.035 \\
\hline$T_{\text {inner }}$ & 16000 & 16000 & 16000 \\
\hline$T_{\text {outer }}$ & 7000 & 7000 & 7000 \\
\hline
\end{tabular}


note that this empirically derived law for the propagation of discrete features has to be regarded as an upper limit; the ambient wind is faster. The stationary wind component was calculated with the SEIBALMER 4 code to $\beta=1.7$ for $\zeta^{1}$ Sco (in Table 3 the $\beta$ values of our target stars are listed along with the pertinent parameters of our wind models (cf. Rivinius et al. 1997)). In passing we note that the radial velocity variations observed by the photospheric lines cause radius variations of the order of $10 \%$ which in turn lead to gravity variations $\Delta \log g$ of about 0.08 dex. The photometric variations of the Strömgren indices (particularly the amplitutes of $c_{1}$ ) of $\zeta^{1}$ Sco can be modeled with ATLAS 8 atmospheres for:

$T_{\text {eff }}=19450 \mathrm{~K}, \log g=2.21, R_{\star}=98 \mathrm{R}_{\odot}$ and

$T_{\text {eff }}=19950 \mathrm{~K}, \log g=2.28, R_{\star}=90 \mathrm{R}_{\odot}$, respectively

This causes luminosity variations of about $10 \%$, which are correlated to the emission height variations. This finding is also important in connection with the wind momentum-luminosity relation of luminous hot stars (Kudritzki, this Volume).

For the nature of the disturbances we favour blobs rather than spherical shells. As shown by Lamers (1994) blobs have a large effect on the profiles if in front of the stellar disk and provide a better explanation of our observations. In fact the profile variability of the absorption components in the hydrogen lines could be reasonably fitted by superposing local density enhancements to the stationary wind (Fig.6).

\section{Conclusions}

The winds of early-B hypergiants are highly variable. The variations are quite different from the wind activity of less luminous BA supergiants (cf. Kaufer, 1998 and this Volume). They are more wind dominated; rotation and corotating interaction regions are not the main effect. Propagating discrete absorption components due to non-spherical density perturbations are typical. The behaviour is similar to the DACs phenomenon in $\mathrm{O}$ stars. The photospheric absorption lines show pulsation-like radial velocity variations which presumably trigger the variations in the wind. Our analysis shows for the first time that disturbances which affect the wind are generated very deep in the atmosphere (i.e. in the photospheric or even sub-photospheric region). The velocity law of disturbances could be followed through a wide range of the wind down to photospheric layers by making use of strategic lines distributed over a wide wavelength range.

The large wavelength coverage of modern spectrographs of high resolution and long-term monitoring programs are the prerequisite for this kind of investigation. 


\section{References}

Appenzeller I., Wolf B., 1979, A\&AS 38, 51

Burki G., Heck A., Bianchi L., Cassatella A., 1982, A\&A 107, 112

Kaufer A., 1998, In: Reviews in Modern Astronomy 11, Schielicke R. (ed.), p. 177

Kiriakidis M., Fricke K.J., Glatzel W., 1993, MNRAS 264, 50

Lamers H.J.G.L.M., 1994, In: Instability and Variability of Hot-Star Winds, Moffat

A.F.J., Owocki S.P., Fullerton A.W., St.-Louis N. (eds.), Kluwer, p.41

Lamers H.J.G.L.M., Snow T.P., Lindholm D.M., 1995, ApJ 455, 269

Prinja R.K., Barlow M.J., Howarth I.D., 1990, ApJ 361, 607

Rivinius Th., Stahl O., Wolf B., et al., 1997, A\&A 318, 819

Schaerer D., de Koter A., Schmutz W., Maeder A., 1996, A\&A 310, 837

Stahl O., Wolf B., Zickgraf F.-J., 1987, A\&A 184, 193

Sterken C., 1977, A\&A, 57, 361

Sterken C., Wolf B., 1978, A\&A 70, 641

Sterken C., Wolf B., 1979, A\&AS 35, 69

Wolf B., Stahl O., de Groot M.J.H., Sterken C., 1981 A\&A 99, 351

Wolf B., Appenzeller I., 1979, A\&A 78, 15

\section{Discussion}

J. Puls: Two types of $\beta$ velocity laws can be found in the literature, namely $v=v_{0}+\left(v_{\infty}-v_{0}\right)(1-1 / r)^{\beta}$ and $v=v_{\infty}(1-b / r)^{\beta}$, where $b$ has to be calculated from $v_{\min } \sim 0.1 \ldots 0.5 v_{\text {sound }}$. If you want to compare observed velocity fields in the lower part with $\beta$ velocity laws (representing the global wind), you should use the latter type since only this parameterisation is consistent with hydrodynamical models.

B. Wolf: Thank you. We will do that in the future. This second velocity law will probably be in better agreement with our observations at the base of the wind.

A. Moffat: How confident can one be that the $\beta$ of the ambient wind $(\sim 1.7)$ is really different from the $\beta$ of the disturbances $(\sim 2.5)$ ?

B. Wolf: We derived $\beta=1.7$ by fitting the profiles of the Balmer lines (mostly $\mathrm{H} \gamma$ and $\mathrm{H} \beta$ ) using a modified version of the SEIBALMER program and the parameters given in Table 3 of my talk. This value provides a substantially better profile fit than $\beta=2.5$.

L. Kaper: The distance to $\zeta^{1}$ Sco (and hence the confirmation of its extreme luminosity) is "known" due to its location in an association, which makes $\zeta^{1}$ Sco a prototype of its class. Is $\zeta^{1}$ Sco's association confirmed (by HIPPARCOS)?

B. Wolf: Yes, it is. $\zeta^{1}$ Sco has a large proper motion. Within the uncertainties $\sigma_{E}=1 \mathrm{mas} /$ year, which corresponds to $10 \mathrm{~km} / \mathrm{s}$ at a distance of $2 \mathrm{kpc}$. The membership of $\zeta^{1}$ Sco in Sco OB 1 is confirmed by HIPPARCOS. 